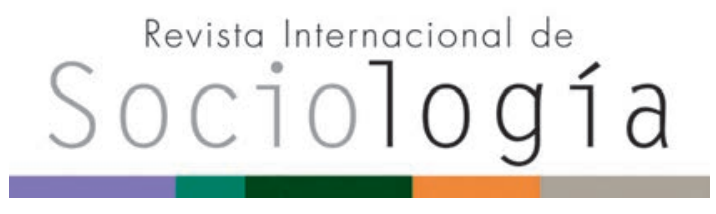

Revista Internacional de Sociología RIS

vol. 76 (3), e103, julio-septiembre, 2018, ISSN-L:0034-9712

https://doi.org/10.3989/ris.2018.76.3.17.01

\section{LOS CUIDADOS EN LA PRÁCTICA. Clase social y estrategias familiares en el cuidado a mayores con dependencias severas}

\author{
TOMÁS CANO \\ Universitat Pompeu Fabra \\ tomasc1010@gmail.com \\ ORCID iD: https://orcid.org/0000-0002-1804-164X
}

Cómo citar este artículo / Citation: Cano, T. 2018. "Los cuidados en la práctica. Clase social y estrategias familiares en el cuidado a mayores con dependencias severas". Revista Internacional de Sociología 76(3): e103. https://doi. org/10.3989/ris.2018.76.3.17.01

\begin{abstract}
Resumen
Esta investigación tiene como objetivo analizar las estrategias familiares de cuidados a mayores con dependencias severas. Para ello nos centramos en las distintas tensiones, condicionantes y justificaciones que se manejan en cada una de las posibles opciones de cuidado, y cómo éstas varían según la clase social. Se usa una metodología etnográfica, mediante la realización de entrevistas en profundidad y observaciones en los hogares de cuatro familias, cada una posicionada en una clase social distinta. El trabajo argumenta que el significado asociado a un "buen cuidado" varía en función de la situación y la clase social. Mientras en la clase alta se justifican las bondades de mercantilizar el cuidado, en las clases más bajas el discurso sobre la estrategia de cuidado a elegir es indeterminado y oscilante. Situaciones excepcionales, como la enfermedad del cuidador principal, justificarían el uso de otras estrategias, como la residencia de mayores.
\end{abstract}

\section{Palabras Clave}

Análisis del discurso; Desigualdad; Envejecimiento; Etnografía; Política social.

\section{CARE IN PRACTICE. Social class and family strategies in the care of elderly people with severe dependencies}

Copyright: (C) 2018 CSIC. Este es un artículo de acceso abierto distribuido bajo los términos de la licencia de uso y distribución Creative Commons Reconocimiento 4.0 Internacional (CC BY 4.0).

Recibido: 04/01/2017. Aceptado: 13/07/2017

\section{Abstract}

This research aims to analyze the different family strategies in elderly people with severe dependencies. To do so we focus in the different tensions, determinants and justifications that are managed in each of the care's possible options, and how these vary across social classes. Ethnography methodology is used, conducting depth interviews and systematic observations in four family's households, each of them belonging to a particular social class. This work argues that the meaning associated to "good care" varies in relation with the situation and the social class. While upper-class families justify the goodness of the care's commodification, in lower-class families the discourses about what care's strategy implement is indeterminate and oscillating. Exceptional situations like the illness of the main caregiver would justify the use of other strategies such as the elderly residency.

\section{KeYWORDS}

Aging; Discourse analysis; Ethnography; Inequality; Social policy. 


\section{INTRODUCCIÓN}

En las últimas décadas varios movimientos sociodemográficos han modificado las estructuras y dinámicas familiares. Entre estos movimientos destacan la masiva incorporación de la mujer al mercado de trabajo, los cambios en las dinámicas familiares (aumento de las tasas de divorcio, familias monoparentales o nuevas formas de convivencia), así como el hecho de que cada vez nacen menos niños mientras que la esperanza de vida aumenta. Este nuevo escenario ha supuesto varios cambios. Por un lado, aumenta la necesidad e intensidad de cuidados a personas mayores dependientes. Por otro lado, se pasa de un modelo principal de cuidados, en el que el trabajo que éstos suponen es realizado por la familia y especialmente por la mujer, a una amplitud de opciones y estrategias de cuidados. Pero ¿qué son los cuidados?

Los cuidados son el trabajo sobre el cual se sostiene la vida (Pérez Orozco 2014). Las actividades que se realizan durante el ejercicio de los cuidados pueden dividirse en dos tipos. Por un lado, las actividades instrumentales, es decir, necesidades materiales como la ayuda económica o tareas como la ayuda con la cocina o la compra. Por otro lado están las implicaciones emocionales, que hacen referencia al conjunto de afectos, comunicaciones o sentimientos que son puestos en juego entre las personas dependientes y los cuidadores durante el ejercicio del trabajo de cuidados (Thomas 2011). Asimismo, los cuidados pueden ser de tipo formal e informal. Los primeros hacen referencia a cuidados de tipo especializado ofertados por profesionales. Los segundos hacen referencia a aquellos cuidados ofrecidos por la red de relaciones de la persona mayor dependiente. Los cuidados informales son realizados de forma voluntaria y no remunerada (Andersson, Levin y Emtinger 2002; Rogero, Prieto y Rosenberg 2008). Los cuidados, asimismo, pueden ser provistos tanto por el Estado - residencias o centros de día, por ejemplo—, como por el mercado — servicios con fines de lucro-, como por la familia — cuidados de hijos a padres, de abuelos a nietos, entre cónyuges, etc. - (Rogero 2009). Por lo tanto, cuando una persona es dependiente puede recibir cuidados de tipo (i) institucionalizados, (ii) mercantilizados o (iii) familiares-informales.

Por su parte, una persona dependiente es aquella que necesita de cuidados para realizar actividades cotidianas y los recibe. La dependencia puede ser de tipo emocional, económico o físico, y puede tener causas de diversa índole, como un accidente, una crisis, el ciclo de vida, la salud o diversidades funcionales (Wilkin 1987). Asimismo, la dependencia puede variar en intensidad. El Instituto Nacional de Estadística, en la Encuesta de Discapacidad, Autonomía personal y situaciones de Dependencia (EDAD), divide entre insignificante, leve, moderada o severa ${ }^{1}$.
Este trabajo se centra en el estudio de los cuidados a personas mayores con dependencia física severa. Con datos de la encuesta EDAD, Abellán, Esparza y Pérez (2011) concluyen que en España hay casi un millón y medio de personas mayores en situación de dependencia, de los cuales la mayoría están en situación de dependencia severa. Asimismo, la tendencia en las últimas décadas es hacia el aumento significativo del número de personas mayores dependientes en situación severa (Abellán, Esparza y Pérez 2011). Éstos dependientes se encuentran en su mayor parte, debido a la desigualdad de género que atraviesa el trabajo de cuidados, atendidos por mujeres, ya sean éstas la pareja de la persona dependiente o su hija (Durán 2000).

No obstante, más allá de los datos cuantitativos, esta investigación se centra en el análisis de los discursos de las familias con una persona dependiente que ponen en práctica una de las tres opciones de cuidados: institucionalización, mercantilización o familiar-informal. El objetivo de esta investigación es doble. Primero, se centra en las constricciones que ejerce la estructura social sobre la elección de una opción de cuidados u otra. Específicamente, se analiza cómo varía la autolegitimación y la definición de "buen cuidado" en las distintas clases sociales, prestando especial atención a las justificaciones sobre las estrategias familiares en torno a los cuidados. Segundo, se observan las desiguales consecuencias asociadas al cuidado de una persona mayor dependiente en relación con la posición de clase ${ }^{2}$.

Esta investigación aporta nuevo conocimiento a la literatura existente en los cuidados a personas mayores dependientes mediante tres vías que han sido poco o nada exploradas previamente. En primer lugar, se centra en el análisis cualitativo de los cuidados a personas mayores con dependencias severas. En segundo lugar, sitúa las diferencias entre clases sociales en el centro del análisis. Finamente, pone en práctica una metodología no utilizada previamente en esta temática como es la etnografía en los hogares y las entrevistas cruzadas entre los miembros de una misma "configuración familiar" (Elías 1987; Martín Criado, Gómez Bueno y Fernández 2000).

\section{ENFOQUE TEÓRICO}

El enfoque teórico del que parte esta investigación se articula con base en dos conceptos principales: habitus (Bourdieu 2007) y don-contra don (Mauss 1979; Godelier 2000). En primer lugar, el concepto de habitus arroja luz a la hora de analizar las significaciones y prácticas en torno a los cuidados en función de la clase social. El habitus remite a las condiciones materiales y simbólicas a las que un individuo (o familia) estuvo expuesto durante su socialización y que condicionarán en buena medida sus gustos, percepciones, hábitos y decisiones a lo largo del cur- 
so de vida. Entendemos en esta investigación que la manera de cuidar el cuerpo enfermo, de atenderlo y mantenerlo, muestra las disposiciones más profundas del habitus: es, en efecto, a través de la compra de los cuidados en el mercado, como pueden enclasarse (es decir, diferenciarse del resto de clases sociales) los grupos sociales dominantes.

En segundo lugar, el concepto de don (Mauss 1979) es definido como el intercambio material o inmaterial y no mercantil que se produce entre individuos de una sociedad. Es la base sobre la que se establece la economía solidaria. Por lo tanto, esta investigación parte de la conceptualización de los cuidados como un conjunto de acciones basadas en el don: el que cuida dona sus recursos - dinero, tiempo, energía- al que es cuidado. El que es cuidado, por su parte, se siente en la obligación de devolver. Pero esta devolución puede tener un valor distinto a lo recibido, así como prolongarse en el tiempo. Por lo tanto, el cuidado, como el don, establece relaciones tanto de solidaridad como de deuda, sobre las que se basa un ciclo de reciprocidad entre el que da y el que recibe. Esto es lo que plantea Tobío (2013), considerando que esta lógica va más allá de un mero intercambio en el que las dos partes implicadas dan y reciben simultáneamente; al contrario, en la lógica del cuidado como don, el dar y el recibir son dos acciones que pueden dilatarse en el tiempo y variar en su contenido material o simbólico.

\section{LA INVESTIGACIÓN RECIENTE EN CUIDADOS A MAYORES DEPENDIENTES Y CLASE SOCIAL}

La investigación reciente sobre cuidados a mayores dependientes, en el caso español, experimentó un gran incremento a raíz de la puesta en marcha de la Ley de Dependencia en 2007. Desde entonces se ha generado un interesante debate tanto teórico (Carrasco 2013; Prieto y Serrano 2013) como empírico (Serrano, Artiaga y Dávila 2013). Esta última investigación concluye que la Ley de Dependencia, más que subvertir la desigualdad de género asociada al trabajo de cuidados, ha contribuido a lo contrario: reproducir una idea de la feminidad asociada a lo informal, lo emocional y lo doméstico. A este respecto, es importante tener en cuenta dos factores. Uno, que esta Ley se pone en funcionamiento en un periodo de recesión económica, lo cual genera un acercamiento al modelo de la austeridad, así como el alejamiento de la convergencia con el modelo social europeo en materia de igualdad (Lombardo y León 2014). Dos: el marcado carácter familista del Estado del Bienestar español (Esping-Andersen 1990). Estos dos factores condicionan tanto el diseño como la aplicación de la ley, tendente a la reproducción de dinámicas previas: aquellas basadas en un modelo dominante de cuidados en el que las personas en situación de dependencia suponen un riesgo de tipo familiar que recae, en mayor medida, sobre las mujeres (Rodríguez-Cabrero 2011).

Sin embargo, para el caso español, son pocos los estudios que se centran en las dinámicas de cuidados a mayores en relación con la clase social. No obstante, existen valiosas excepciones. El estudio de Castelló (2011) desarrolla el concepto de habitus de cura con objeto de explicar los condicionantes que están detrás de la división sexual del trabajo de cuidados o -lo que es más importante-qué lleva a optar por una opción de cuidados u otra; es decir, a elegir entre el Estado, el mercado o la familia. Esta investigación concluye que las mujeres de clase trabajadora desarrollan estrategias de cuidados informales en el hogar, haciendo uso de otras opciones, como la institucionalización o la mercantilización, cuando los recursos familiares no están disponibles. Mientras, las mujeres de clase media desarrollan modelos mixtos basándose en la familia, la institucionalización y, en menor medida, la mercantilización. Finalmente, las mujeres de clase alta optan por la mercantilización, ejerciendo cierta resistencia a la institucionalización. Los resultados de investigaciones de tipo cuantitativo parecen corroborar estos resultados. Estos trabajos concluyen que el nivel educativo, uno de los indicadores de clase social más utilizados en la literatura, se relaciona con el modelo de cuidados: a mayor nivel educativo, mayor probabilidad de recibir más cuidado formal y menos cuidado informal (Rogero 2009; 2010; Otero et al. 2003). Martin y Vilaplana (2012) sugieren que esto puede deberse al coste de oportunidad: conforme más alta es la posición social de la familia, mayor es el coste de renunciar a un empleo para realizar cuidados informales en el hogar. Asimismo, Rogero (2009) concluye que la opción de recibir cuidados de tipo formal como única fuente de apoyo es una opción condicionada a la disponibilidad de recursos socioeconómicos.

Por su parte, Martinez-Buján (2008), en su investigación centrada en la mercantilización del cuidado, considera que esta opción depende más de la implicación de la mujer en el mercado laboral que de la clase social de familia. Es decir, no se trata tanto de una cuestión de clase sino de disponibilidad temporal. Asimismo, esta investigación considera que la preferencia familiar sobre la contratación de mujeres, preferiblemente suramericanas, para realizar el trabajo de cuidados, se basa en el estereotipo de que son ellas las que tienen mayor capacidad de "paciencia" y de "cariño". Por lo tanto, cuando se elige esta opción se obvian los requisitos profesionales de las cuidadoras, exaltando dichas capacidades. Esta autora encuentra que en ellas se delega tanto el cuidado más instrumental como el emocional. El estudio de Moré (2015) también se centra en la mercantilización del cuidado, desde la perspectiva de las mujeres que son contratadas y a las cuales se les delega 
el cuidado de mayores. Esta investigación considera que las mujeres contratadas para cuidar otorgan sentido a sus labores a través de las emociones, terminando por considerar a los mayores cuidados como su propia gente. Sin embargo, Martínez-Buján (2008) apunta que este "cariño" sería ficticio, puesto que está basado en una relación salarial, y solo el paso del tiempo posibilitaría una relación íntima entre cuidadora contratada y persona dependiente.

\section{Metodología}

\section{Técnicas de recogida de información y trabajo de campo}

La práctica más habitual en la investigación sociológica cualitativa mediante entrevista consiste en ir al terreno y entrevistar a una serie de personas, a través de cuyo discurso consideramos teóricamente que llegaremos a la realidad de su vida cotidiana. Esta investigación, sin embargo, representa un trabajo etnográfico: acudimos a los hogares investigados, convivimos con ellos y entrevistamos a los miembros de las familias que mayor responsabilidad en el cuidado; manteniendo también distintas conversaciones con allegados de las familias. Esto nos ha dado la posibilidad de ir más allá de los discursos, recogidos en las entrevistas, confrontándolos con las prácticas recogidas a través de la observación.

Haciendo varias entrevistas cruzadas en cada una de las familias seleccionadas fue posible observar y analizar el conjunto de tensiones, conflictos y cooperaciones surgidas en el seno familiar en torno al empleo y el trabajo de cuidados, así como los juegos internos de poder y dominación. La realización de entrevistas cruzadas en un mismo seno familiar es una cuestión fundamental: los individuos saben mucho acerca del entorno en el que viven y trabajan. No hay nadie que sepa más sobre la práctica cotidiana del cuidado que el propio cuidador y las personas con las que convive. Así que una vez nos adentremos en el hogar y conversemos con cada uno de sus miembros, obtendremos información valiosamente contrastada. Ya que, como explica Becker (2009: 133): "Yo no sé nada que alguien de ese grupo no sepa, pero, dado que sé lo que todos saben, sé más que cualquiera de ellos".

El trabajo de campo se realizó durante 2011 y 2012 en las localidades de Linares y Úbeda (Jaén, España). Andalucía es una comunidad autónoma especialmente interesante para el estudio de los cuidados, debido a que combina un elevado familismo con un elevado nivel de subvención: el $30 \%$ de los mayores cuentan con una prestación económica para la dependencia y el $77 \%$ son cuidados por algún familiar (Martínez-Buján 2014). Esta misma autora denomina el caso andaluz como "modelo familista subvencionado” (Martínez-Buján 2014: 115).
El trabajo de campo tuvo una duración total de nueve meses, durante los que se realizaron notas, fotografías y grabaciones de entrevistas en el seno de los hogares. El material empírico fue transcrito y analizado mediante el método de análisis sociólogo del discurso (Martín Criado 1998; 2014; Conde 2009).

\section{Selección de la muestra}

Se seleccionaron cuatro casos. Cada uno equivalía a una familia de diferente clase social. Así, se realizó un muestreo estructural, donde cada caso buscaba representar — no estadísticamente- - un universo discursivo vinculado a una posición de clase (Ibáñez 1979).

La variable clase social se operacionalizó de la siguiente manera: a) clase alta, familias donde las personas entrevistadas tuvieran estudios universitarios, elevado nivel patrimonial y posiciones de dirección en el mercado de trabajo; b) clase media alta, con estudios universitarios y mandos medios en el mercado de trabajo; c) clase media baja, con estudios de FP o bachillerato, trabajadores de cuello blanco; d) clase baja, con estudios básicos, desempleados temporales con incursiones intermitentes al mercado de trabajo.

Investigaciones previas (Rogero 2009; 2010) establecen una asociación positiva entre clase social y opción de cuidados: a mayor nivel socio-económico, mayor probabilidad de recibir cuidado formal; y viceversa, a menor clase social mayor probabilidad de recibir cuidado informal. La selección de casos de esta investigación buscó ajustarse a la asociación positiva entre los continuos de clase social y formalidad-informalidad encontrada en investigaciones previas. Así, en el caso de clase alta el cuidado es mercantilizado veinticuatro horas (totalmente formal), siendo este grado de formalidad decreciente según se desciende de clase social.

Asimismo, se cuidó la homogeneización de casos en su selección, en función de una serie de factores que, según han demostrado estudios previos, afectan las preferencias y estrategias de cuidado más allá de la clase social. Primero, la selección se restringió a casos de dependencia severa por enfermedad no sobrevenida. Esto es relevante, ya que una de las principales variables que condicionan las preferencias y estrategias de cuidado es el nivel de gravedad de la dependencia (Fernández-Carro 2016). Segundo, dado que existen diferencias significativas en los modelos de provisión de ayudas sociales a los cuidados, no solo entre países, sino también entre comunidades autónomas (MartínezBuján 2014), todos los casos se encontraban en la misma comunidad autónoma. Tercero, todas las familias pertenecían a un medio semiurbano y convivían en localidades menores de 100.000 habitantes pero mayores de 30.000. Cuarto, todas eran familias "intactas", es decir, no hubo divorcios, separa- 
ciones o relaciones familiares especialmente conflictivas, ya que un elevado conflicto familiar puede llevar a reducir el deseo de mantener el cuidado informalmente en el hogar (Fernández-Carro 2016). Quinto, en ninguna de las configuraciones familiares había un segundo familiar con dependencia severa, lo cual se relaciona con una mayor probabilidad de externalización de los cuidados, ya que supone una elevada carga de trabajo informal para los familiares (Spijker y Zueras 2016). Sexto, ninguna de las familias ha sido gravemente afectada por la recesión económica de 2007. Finalmente, se trata de familias donde no ha habido movilidad geográfica entre generaciones. Esto también es relevante porque una familia dispersa geográficamente puede generar una mayor preferencia hacia la institucionalización o mercantilización del cuidado, como observó Martinez-Buján (2008), ya que en estas familias también aumenta el coste de oportunidad de cuidar al tener que desplazarse geográficamente.

\section{Descripción de los casos}

El primer caso representa la configuración familiar de clase alta. La persona dependiente es Juana, de 81 años, padece demencia senil y es viuda. Tiene dos hijos varones, Mateo y Luís, de 58 y 56 años respectivamente, y con los cuales me entrevisto (ver Tabla 1). Ambos están casados y tienen hijos. La persona dependiente vive en su propio hogar junto con dos mujeres extranjeras que se turnan para atenderla las 24 horas del día. Por lo tanto, ponen en práctica la estrategia de mercantilización de los cuidados en el hogar. Las cuidadoras formales son suramericanas, madre e hija, tienen un salario de $1.000 €$ netos cada una y libran un día a la semana, el domingo. Cada domingo, Mateo y Luis se turnan para acudir al hogar de su madre mientras las empleadas descansan. Tanto la mujer de Mateo como la de Luis colaboran activamente en el trabajo instrumental de cuidados: hacen compras en el supermercado, preparan comidas, etc. Juana es beneficiaria de la ayuda económica de la Ley de Dependencia, la cual usan para cubrir parte del salario de las mujeres contratadas.

El segundo caso representa la configuración familiar de clase media-alta. La persona dependiente es Pedro, de 82 años y padece de alzhéimer. Vive con su mujer Carmen, de 79 años, y con unas condiciones de salud favorables, por lo que es independiente para realizar actividades básicas de la vida diaria. Pedro y Carmen tienen dos hijos casados y uno viudo viviendo en la misma localidad, y un cuarto emigrado al extranjero. Me entrevisto con Carmen y con uno de los hijos y tengo conversaciones con las cuatro nietas y los dos nietos. Este es el caso más dinámico de los estudiados en cuanto a estrategias de cuidados. Justo antes del comienzo del trabajo de campo, Pedro estaba en su hogar, con Carmen como cuidadora principal y una mujer que acudía dos horas al día para ayudar a Carmen en las tareas de cuidado físicamente más demandantes, como levantar o acostar a Pedro. Al comienzo del trabajo de campo la familia decide internar a Pedro por las mañanas en un centro de día. El resto del día lo pasa en su hogar con su mujer. Sin embargo, dos meses después pasa a estar veinticuatro horas internado en una residencia pública de mayores. Por lo tanto, se mueven desde la mercantilización combinada con cuidado familiar-informal hacia la institucionalización del cuidado. Carmen y sus cuatro hijos son los principales encargados del cuidado, en el que también colaboran dos de las mujeres y las cuatro nietas. Estas tienen edades comprendidas entre los dieciocho y los veintiocho años y son enfermera, profesora, carnicera y estudiante de enfermería respectivamente.

El tercer caso representa la configuración familiar de clase media-baja. La persona dependiente es María, de 60 años. Padece de una enfermedad degenerativa severa en estado avanzado desde hace seis años. Su marido, Manuel, tiene 64 años. Están casados y tienen dos hijos: Violeta, de 31 años, y Luis, de 35. María está veinticuatro horas en su hogar donde vive con su marido Manuel y es cuidada por él. En el hogar también viven su hija Violeta y, algunos meses del año, Marga, de 57 años y hermana de María. Me entrevisto con Manuel, Marga ${ }^{3}$ y Violeta. Manuel y María viven de sus pensiones, a lo que suman la prestación económica de la Ley de Dependencia. Esta prestación se destina a contratar a una mujer que ayuda con cuidados de tipo instrumental, como colaborar en la limpieza del hogar y en las compras de comida. Esta familia realiza cuidado informal en el hogar y externalizan —-mercantilizan — una pequeña porción de cuidados de tipo instrumental.

Finalmente, en el último caso, de clase baja, se realizan la totalidad de los cuidados en el hogar. La persona dependiente es Iñaki, de 82 años, viudo y enfermo de alzhéimer. Iñaki tuvo que mudarse hace dos años al hogar de su hija Toñi, de 54 años, donde también viven el marido de Toñi, Paco, de 61 años y prejubilado; María, hermana de Toñi e hija de Íñaki, y el hijo que tienen en común Toñi y Paco, Antonio, de treinta años, que vive intermitentemente en el piso mientras busca empleo. En este caso se realizan cuidados informales en el hogar. Toñi, sin ingresos y "cuidadora veinticuatro horas", vive gracias a las pensiones de su padre, de su marido y a la ayuda de la Ley de Dependencia.

Las estrategias familiares que se ponen en práctica en los distintos casos son de carácter dinámico, es decir: las familias van utilizando y ajustando sus recursos disponibles para atender a la persona dependiente en función de la situación. En los resultados que a continuación se presentan se expone cada una de las estrategias por separado. Esto no quiere decir que en algunos casos no se haga uso de otras estrategias simultánea o secuencialmente, como se explica en las descripciones de los casos. 
Tabla 1.

Información detallada de las personas entrevistadas en cada uno de los casos analizados.

\begin{tabular}{|c|c|c|c|c|c|}
\hline $\begin{array}{l}\text { Configuración } \\
\text { familiar }\end{array}$ & Nombre $^{*}($ Edad $)$ & Nivel educativo & Posición laboral & Ingresos mensuales & $\begin{array}{l}\text { Relación con } \\
\text { la persona } \\
\text { dependiente }\end{array}$ \\
\hline \multirow[t]{2}{*}{ Caso 1} & Mateo (58) & Licenciado & $\begin{array}{l}\text { Directivo en Adminis- } \\
\text { tración pública }\end{array}$ & Entre $2.000 €$ y $3.500 €$ & Hijo \\
\hline & Luis (56) & Diplomado & $\begin{array}{l}\text { Directivo empresa } \\
\text { privada }\end{array}$ & Más de $3.500 €$ & Hijo \\
\hline \multirow[t]{2}{*}{ Caso 2} & Carmen (79) & Primarios & Jubilada & Menos de $1.000 €$ & Pareja \\
\hline & Juan (59) & Doctorado & $\begin{array}{l}\text { Directivo en Adminis- } \\
\text { tración pública }\end{array}$ & Entre $2.000 €$ y $3.500 €$ & Hijo \\
\hline \multirow[t]{3}{*}{ Caso 3} & Manuel (62) & $F P$ & Pre-jubilado & $<1.000 €$ & Pareja \\
\hline & Marga (57) & $F P$ & Auxiliar enfermería & Entre $1.000 €$ y $2.000 €$ & Hermana \\
\hline & Violeta (31) & $F P$ & Peluquera & $<1.000 €$ & Hija \\
\hline \multirow[t]{3}{*}{ Caso 4} & Toñi (54) & Primarios & Inactiva & Sin ingresos. & Hija \\
\hline & María (49) & Primarios & En paro & $<1.000 €$ (Subsidio desempleo). & Hija \\
\hline & Antonio (30) & Secundarios & En paro & $<1.000 €$ (Subsidio desempleo). & Nieto \\
\hline
\end{tabular}

*Nota: Todos los nombres son seudónimos.

\section{Clase social y estrategias FAMILIARES EN EL CUIDADO A MAYORES DEPENDIENTES}

\section{La Institucionalización del cuidado}

La residencia de ancianos es un "desierto de soledad", decía Norbert Elias en La soledad de los moribundos (1987). Así es percibido por los familiares de los dependientes, sin distinción de clase. Desde las clases más altas a las más bajas, internar a un familiar enfermo en una residencia de mayores (institucionalización del cuidado) sería una práctica connotada negativamente y acompañada, en la mayoría de los casos, de cierta resistencia por parte del propio dependiente:

Mateo: "Alguna tentación tuve yo [de internarlo en una residencia], pero llegamos al acuerdo de que era mejor que estuvieran en su casa. Además mi padre, que siempre ha tenido más conciencia, así lo quería."

(Caso 1, clase alta).

En el segundo caso, de clase media-alta, el único que recurre a la institucionalización durante las veinticuatro horas, una vez el alzhéimer entra en un estado avanzado. En las entrevistas y observaciones realizadas en este caso se justifica la decisión de internar a la persona dependiente porque, según consideran, se encuentran "en una situación límite" por distintos motivos (Juan, clase media-alta). En primer lugar, Carmen siente que está demasiado mayor tanto para cuidar de su marido en estado avanzado de alzhéimer, como para asumir la convivencia continuada en su propio hogar con cuidadoras contratadas ajenas a la familia. En segundo lugar, los hijos, todos varones y trabajando a jornada completa, se enfrentan a una decisión racional con base en un ele- vado coste de oportunidad. En tercer lugar, solo dos de las mujeres de éstos se encuentran en la misma localidad: una ya cuida de su propia madre dependiente; la otra trabaja a tiempo completo. Finalmente, en esta "situación límite", entre las nietas se plantea ${ }^{4}$ la posibilidad de renunciar al empleo para cuidar a su abuelo. En ese momento salen a flote tensiones de género que pivotan sobre la posible legitimidad de esta decisión, generándose dos polos discursivos. Uno mayoritario de orientación al empleo que, no sin tensiones, subordina las funciones reproductivas a las productivas, así como lo comunitario a lo individual: el proyecto laboral sería lo primero. Otro minoritario donde se continúa percibiendo el cuidado como el espacio "natural" de la mujer. Finalmente, la agudización de la enfermedad, combinada con la negación de Carmen sobre la posibilidad de convivir con cuidadoras contratadas, sumado a un discurso legítimo con base en el desarrollo personal y laboral, así como la disposición de recursos suficientes para costear una residencia, terminan decantando la balanza a favor de la institucionalización del cuidado.

Es interesante destacar en este punto la desigualdad de género. Mientras que entre las nietas se generan tensiones y discusiones sobre qué sería lo más legítimo, en el caso de los hijos varones esa posible duda ni se plantea: para ellos solo hay un rol legítimo, el de "ganadores del pan". Finalmente, los nietos, durante las tensiones y discusiones sobre qué estrategia de cuidados tomar, ellos desaparecen, tanto de los discursos como de las prácticas de cuidados.

Una vez se ha tomado la decisión, aparecen las justificaciones sobre la institucionalización del cuidado. En estos discursos se disocia entre las cualidades técnicas y las cualidades afectivas de las residencias. En los momentos en los que la decisión familiar 
pasa por internar al dependiente en una institución — pública o privada-, las justificaciones discursivas dejan aflorar los argumentos que ensalzan las cualidades técnicas de las residencias y sus recursos humanos: "Ahí hay enfermeras y trabajadores muy bien cualificados" (Carmen, clase media alta); y se eluden -en la medida de lo posible - las carencias afectivas que se asocian con las residencias. Supone, en cierto modo, un mecanismo de evasión de la culpa, dado que el uso de la residencia, una estrategia con cierta connotación negativa, puede producir remordimiento en las familias. Así, la norma social se muestra interiorizada en forma de culpa.

Significados diferentes, aunque no radicalmente opuestos, se atribuirían, en los casos de clase mediabaja y baja, a la institucionalización del dependiente en un centro. Aquí la indeterminación sobre esta estrategia de cuidados es constante. El discurso vuelve a oscilar entre la posible legitimidad y la posible sanción social asociada al hecho de internar a un mayor en una residencia. Por tanto, la pregunta iría en esa dirección: ¿Cuándo sería legítimo, en posiciones sociales desaventajadas, ingresar en una residencia a un familiar mayor enfermo? Las disputas sobre la legitimidad de valerse de los servicios de una residencia son constantes y los límites fluctúan incesantemente: se culpa a otros familiares de lo que uno mismo reconoce hacer en otro momento, se afirma y se niega en diferentes momentos la virtud de la residencia:

Toñi: "Yo decidí cuidar a mis padres cuando estaban enfermos porque no era capaz de meterlos en una residencia. Si hubiera sido capaz, a mi padre y a mi madre era como abandonarlos. Cuando mi madre empezó con el alzhéimer, yo psicológicamente no era capaz de meterla en una residencia." (Caso 4, clase baja)

Pero lo dicho, más tarde, se contradice con lo hecho:

Toñi: "Metí a mi padre después en una residencia. Lo decidí más que nada por mi salud. Necesitaba ese tiempo para mí y en esas circunstancias es que no podía atender ni a mi marido ni a mi padre. Ese fue el motivo por el que lo ingresé en la residencia." (Caso 4, clase baja).

Los marcos mentales a los cuales se asocia la residencia son términos como "abandono" del dependiente o "desidia" familiar. Por tanto, si se optara por dicha estrategia, como en el caso anterior, la justificación de la práctica concreta sería inminente y reiterativa. En los casos de clases más bajas analizados, solo una cosa podría justificar esta acción: la enfermedad. Ya sea esta sufrida en la piel del cuidador, que legitimaría el internamiento del dependiente a su cargo así como el cuidado de sí mismo; ya sea sufrida en la piel del dependiente, que de nuevo justificaría su internamiento. Si el motivo que justifica el internamiento fuese el agravamiento del estado de salud de la persona mayor dependiente, en este caso se exaltarían los discursos asociados con las incapacidades técnicas del hogar para prestar cui- dados a enfermedades graves, ocultando o desplazando a un segundo nivel las virtudes afectivas del cuidado en el hogar.

Para cerrar este epígrafe observemos el caso de una mujer de clase desaventajada y sus esquemas de legitimidad frente a la residencia. Es el caso de María. En su narrativa adopta una posición discursiva de buena hija, que jamás abandonaría a una madre (o padre) para internarlos en una residencia. Atiende al espacio semántico del amor y el afecto de una madre a una hija, que debe ser devuelto en forma de un contra don; "tú me cuidaste cuando yo era pequeña y dependiente; ahora que tú eres mayor y dependiente, yo te cuidaré". Como diría Mauss (1979), lo que obliga a cuidar es el hecho de que el cuidado obliga: si se fue cuidado en la infancia, se siente la obligación de devolver esa donación en la vejez. Este es un esquema cognitivo incorporado en las mujeres adultas de clases medias y bajas analizadas, es un esquema legítimo del cuidado basado en el don-contra don.

\section{La mercantilización del cuidado en el hogar}

A continuación se analiza la estrategia denominada mercantilización del cuidado en el hogar. Sennet y Cobb (1973) consideraban que las personas que se ubican en posiciones dominantes de la estructura social exhiben sus credenciales simbólicas (nivel educacional, por ejemplo) o materiales (patrimonio, por ejemplo) como respuesta y correlación de prácticas sociales como, en este caso, la elección de una estrategia de cuidado u otra. Luis (clase alta), enlazaba así sus recursos materiales con la estrategia de cuidados elegida: "Por suerte tengo patrimonio suficiente para contratar a dos mujeres". Así, la autoasignación de mayor valía social les hace merecedores de un reconocimiento que justifique la desvinculación del trabajo doméstico o de cuidados; trabajo delegado en otros y, sobre todo, en otras.

Luis: "Hemos tenido mucha suerte en las mujeres que atienden a mis padres traspasando lo puramente laboral y pasando a un nivel familiar (...). Mi experiencia con ellas ha sido excelente. Son suramericanas, suelo hablar con ellas -voy todos los días- y tienen las cosas muy claras. Para mí es la mejor opción... se lo suelo decir a todo el mundo." (Caso 1, clase alta).

A pesar de la dominación propia de la relación empleador-empleada, el tiempo y la buena relación entre ambos motiva esa combinación de términos laborales y personales. El control continuado sería la vigilancia sobre la buena praxis ejercida por la cuidadora contratada sobre la persona mayor dependiente, también como mecanismo para evitar posibles conflictos con la cuidadora. Si una situación de conflicto sucediera, supondría una carga de trabajo extra para los responsables del cuidado de la persona mayor; carga que habría que intentar soslayar en todo momento. 
Por lo tanto, este caso concreto, representativo de una tipología de cuidados muy extendida - sobre todo previamente a la crisis económica- entre familias con elevados niveles educativos (Rogero 2009), se sustenta sobre la interrelación constante de tres actores en juego: la persona mayor dependiente, su familia y la o las cuidadoras contratadas. Las afecciones asociadas a las enfermedades severas reconfiguran la capacidad de la persona mayor de interactuar en el entramado de relaciones entre los tres agentes. La relación entre empleador y empleada se encuentra sustentada sobre la base de la dominación de empleador a empleada y la lógica de la interdependencia entre ambos.

En dicha dominación de empleador a empleada no es baladí que las personas contratadas para realizar el cuidado sean mujeres y extranjeras. Cabe resaltar que el trabajo de cuidados representa una de las actividades sociales donde la desigualdad de género es mayor (Folbre 2007; Durán 2000; Martín-Palomo 2013). Asimismo, Rollins (1985), para el caso de Estados Unidos, observó que las clases altas, a la hora de externalizar el cuidado, tienen preferencia por las mujeres extranjeras ya que éstas son percibidas como más dóciles, por tener interiorizado el ejercicio de la sumisión. Martínez Buján (2008) encontró similares dinámicas para el caso español.

El caso de clase alta analizado es el único que pone en práctica esta opción de cuidados. En los discursos analizados en esta configuración familiar los mecanismos de atribución de valor social varían respecto a casos de posiciones sociales más bajas. Aquí no se atribuye el valor social al buen hijo en términos de duros trabajos físicos de sometimiento a las labores de cuidados, sino en términos intelectuales, de conocimiento y preocupación sobre el proceso de atención. En el caso de la clase alta no se deslegitimaría al que elude el cuidado por razones laborales u otras, sino al que no visita, al que no conoce de primera mano y puntualmente la situación y afección de la persona dependiente:

Mateo: "Sé de uno [habla de un amigo] que, si le preguntaba por su madre, que la tuvo en la residencia,

él no sabía cómo se encontraba, si estaba mejor o si estaba peor (...)" (Caso 1, clase alta).

Como vemos en el verbátim previo, como en todos los grupos sociales, las prácticas de cuidado son juzgadas por el grupo de pares. Aquí el discurso proyecta una imagen de sí mismo vinculada a la de "buen hijo", a través de la oposición a lo que se consideraría un "mal hijo": aquel que no solo no cuida, sino que tampoco visita a la persona dependiente, ni controla a los detentadores del cuidado (enfermeras y auxiliares de la residencia, mujeres contratadas, etc.), ni conoce ni se informa de las cuestiones teóricas y prácticas asociadas a la enfermedad. Para Mateo, miembro de la configuración familiar más aventajada socioeconómicamente, el "buen hijo" es riguroso e informado. Por lo tanto, él se autodisciplina para realizar visitas cotidianas y saber cada día y de primera mano la situación de su madre enferma, así como para controlar a las mujeres que tiene contratadas y sobre las que recae la carga del trabajo: "Me lo propuse como una actividad en mi calendario y tengo que cumplirlo", me decía.

Esta opción de cuidados, la mercantilización en el hogar, debido a su elevado coste, es una opción difícilmente viable para el caso de clase baja. Como se observó en la descripción de los casos, en este hogar los recursos económicos son demasiado bajos para afrontar esta posibilidad. A las constricciones económicas hay que sumar las constricciones simbólicas:

\begin{abstract}
Toñi: "A mí no me gusta. Tienes que tener mucha suerte para que tengas a una persona y trabaje como yo considero que tiene que trabajar. De diez, ocho no están contentas. Son árabes, suramericanas... y yo no sé si ellas saben lo que es el alzhéimer. No es para mí una opción válida. Estás metiendo a alguien extraño en tu casa. Me da miedo (...). Imagínate en la casa... que cuando estas delante, bien, pero ¿y cuando no estas delante? Además, todos perdemos los nervios. Es a ti y te agotan la paciencia, imagínate a alguien que no les duele. Yo respeto a quien lo decida, pero para mí no es una opción." (Caso 4, clase baja).
\end{abstract}

Toñi justifica la no elección de esta opción a través de la deslegitimación de las cuidadoras externas. Las mismas mujeres que en el caso de clase alta son alabadas por su buen hacer, aquí siembran el miedo y la desconfianza.

Mientras que las dos posiciones más extremas caso de clase alta y caso de clase baja- legitiman el uso o no uso de la misma opción de cuidados de forma enfrentada, en los casos de clases medias no se encuentra tal disputa por la legitimidad de su uso y se elige esta opción tan solo como refuerzo de cuidados. Este refuerzo puede venir en varias formas: como ayuda extra a los cuidados realizados en el hogar - para apoyar con las tareas domésticas, la compra, cocinar, etc.- en combinación con otras opciones de cuidado - centros de día, por ejemploo en momentos que se requieren cuidados de tipo puntual que exceden o cargan físicamente de manera excesiva a cuidadores principales -levantar al dependiente de la cama, acostarlo, etc.-.

\section{El cuidado informal en la familia}

¿Por qué en unos casos se mantiene el cuidado en el hogar mientras en otros se mercantiliza o institucionaliza? A continuación podemos observar los motivos que evitaron mantener el cuidado informalmente en el hogar en el caso de clase alta analizada:

Luis: "No hemos querido [cuidar informalmente a su madre]. Y no hemos querido tácitamente. Mi hermano y yo (...) no nos hemos querido comprometer con 
el rigor de todos los días del año a la misma hora... para acostarla, para todo... Digo tácitamente porque esto no lo hemos hablado. (...) Pues si no hubiéramos tenido un buen patrimonio, pues no hubiera sido así, pues no lo sé, no sé cómo hubiera sido... ¿Dos mujeres? [refiriéndose a las dos mujeres que tienen contratadas] Pues probablemente no. Que nosotros no tengamos esas responsabilidades, todos los días, a la misma hora estar para levantarla y para acostarla y cuidarla... Pues a lo mejor nos hubiéramos visto obligados a hacerlo, claro está." (Caso 1, clase alta).

La intersección de la clase social con el género en el caso 1, como en el caso 2, los hijos son varones y de posición social media-alta-, sumado a la preferencia de la persona dependiente de quedarse en su hogar, terminan condicionando la decantación hacia la mercantilización de los cuidados. Los hombres de clase alta juegan con una ventaja comparativa a la hora de tomar decisiones sobre qué opciones tomar. Se tienen recursos suficientes para contratar a dos mujeres externas a la familia a jornada completa; ni tan siquiera se plantea la posible opción por parte de los hijos de renunciar a sus empleos para dedicarse al cuidado: el coste de oportunidad es demasiado alto. Asimismo, la construcción social del género sustenta dicha elección y reduce posibles remordimientos y culpas que, en cualquier caso, apenas existen pues sus "padres así lo querían" (Mateo, clase alta).

Por contra, en los casos de clases bajas los motivos que llevan a privarse de una vida en la esfera pública, autónoma social y económicamente, o a prescindir del sentido del disfrute, de las relaciones sociales o del trabajo remunerado en el mercado, viene impuesto por una escasez de recursos que lleva a las mujeres de clases populares analizadas a una vida entregada al cuidado. El escaso volumen de capitales y la posición desfavorecida que ocupan en la estructura social, incorporan en los habitus de clase una forma de percibir otras opciones que no sean el cuidado informal en el hogar, no solo como algo materialmente inalcanzable, sino como una práctica que desvaloriza, que se opone a lo legítima y socialmente aceptado por los grupos sociales en parecidas o similares posiciones sociales: "Las prácticas de todos los agentes de una misma clase, deben la afinidad de estilo" (Bourdieu 2012: 203).

En los dos casos estudiados que representan posiciones sociales más desfavorecidas el cuidado se mantiene informalmente en el hogar. En estos casos, una de las cuestiones más repetidas en torno a la significación de los cuidados es la dureza y la intensidad que suponen este tipo de trabajos. La atención de un dependiente severo en el hogar implica una servidumbre constante, es decir, pesa sobre el cuidador principal sin descanso, día y noche, sin interrupción ni tregua; no existe una definición del tiempo, la vida pasa a ser una jornada laboral continua, perpetua e inevitable:
Manuel: "Soy el que está diariamente con ella los 365 días del año. No me separo de ella nunca” (Caso 3, clase media-baja).

Marga: "Me iba a entrar depresión ¿sabes? Y es muy duro, es muy duro. Esto... no hay dinero para pagarIo" (Caso 3, clase media-baja).

Antonio: "Esto es todo el día, todo el día, las veinticuatro horas" (Caso 4, clase baja).

Toñi: "El cuidado del enfermo de alzhéimer es de veinticuatro horas al día y no tienes ni tiempo para peinarte muchos días" (Caso 4, clase baja).

En estos casos, el cuidador dejaría todo, incluso a sí mismo, para dedicarse "en cuerpo y alma" a la persona dependiente. Debido al hecho de que el tiempo es un juego de suma cero - si se dedica mucho tiempo al cuidado se debe eliminar tiempo de otras esferas de la vida, como el empleo o el ocioy el cuidado a la dependencia severa requiere de atención continuada y permanente, ninguno de los cuidadores principales, en los casos de clase baja y media-baja analizados, tienen un empleo remunerado. Compaginar los cuidados de larga duración y el empleo sería temporalmente inviable. El cuidado a este tipo de dependencia implica actividades física y emocionalmente demandantes, e inflexibles, que explican la dificultad de compaginar con un empleo. Esta situación conlleva diversas consecuencias adversas para estas configuraciones familiares.

La primera de las consecuencias es la disminución —o la imposibilidad de aumento- de capital económico, debido a las dificultades de acceso al mercado de trabajo remunerado. Sus condiciones materiales de existencia quedan delegadas y a expensas de un Estado benefactor. El agravamiento progresivo de la enfermedad y la consecuente discapacidad va acompañándose de una disminución, igualmente progresiva, de las relaciones sociales extrafamiliares de los cuidadores, esto es, de su capital social. Los cuidadores de los casos de clase media-baja y baja prácticamente han perdido su red social y, por lo tanto, el capital que esta les puede proporcionar. Tan solo mantienen unas cuantas amistades con otras personas que se encuentran en la misma situación, es decir, que son cuidadores:

Manuel: "Aquí los amigos venían cuando había lirili... ahora que no hay lirili no vienen ni de visita..." (Caso

3, clase media-baja)

La adaptación a la vida de cuidador principal de una persona mayor dependiente constituye un mecanismo de asimilación subjetiva a unas condiciones objetivas de vida que imponen el sufrimiento como la lógica que estructura su comportamiento. A veces, readaptarse o encontrar el sentido a esta especie de "vida carcelaria" 5 puede acarrear una búsqueda que dure toda una vida — la del dependiente-. La esperanza de la vuelta a la esfera púbica o al trabajo remunerado pasa por dos opciones amargas: 
el internamiento en un centro del dependiente - que es económicamente inviable- o el fallecimiento del mismo -cuestión que, con solo pensarla, abre la puerta a todo un mundo de nuevos significados, incertidumbres, pesadumbres y sinsentidos-:

\begin{abstract}
Manuel: "Pues al final tuve que pensar y resetearme y decir, bueno, aquí esto es un problema que lo tengo que asumir yo que soy su marido y la ayuda que nos pueda dar el resto de la familia, la mucha o la poca. Y tuve que decir, bueno, si yo me vengo abajo, ¿quién va a cuidar de mi mujer? Que hay una cosa que yo siempre tengo encima de mí que es que yo no me ponga enfermo, es que, si yo me pongo enfermo, mi casa se hunde" (Caso 3, clase media-baja).
\end{abstract}

\section{Variaciones en consecuencias y reconoci- mientos simbólicos según clase y género}

En la intensidad y dureza del trabajo que supone cuidar nos encontramos con una de las situaciones -y sus consecuencias - en que la clase social opera de manera más intensa. Mientras las personas pertenecientes a clases bajas soportan un hacinamiento continuo, con los efectos que dicha situación conlleva, el caso contrario lo ocupan las familias más acomodadas. En estas configuraciones familiares de clase alta los términos dureza, intensidad o servidumbre son sustituidos por control, supervisión, visitas o autodisciplina - "atractores centrales", usando la terminología metodológica de Conde (2009)Las cargas de trabajo que supone el cuidado de dependencias severas -que sería el hilo discursivo- es comprado en el mercado, ya sea mediante el internamiento de la persona mayor en residencias -normalmente privadas - o contratando a cuidadores —normalmente cuidadoras y extranjeras-.

La dependencia severa de un mayor tiene consecuencias dispares sobre la riqueza y capitales de las configuraciones familiares analizadas. Una vez que uno de los miembros de la familia cae enfermo, la posición social de partida es determinante para atenuar o multiplicar las consecuencias socioeconómicas sobre la configuración familiar. En los casos de clase media-alta y alta, al ser la mayor parte del cuidado delegado a otros miembros externos a la familia, el tiempo disponible y, en efecto, los capitales, se ven afectados en menor grado. Lo contrario ocurre en los casos de clase media-baja y baja. En estos últimos, al tener menos recursos de partida y enfrentar dificultades materiales y constricciones culturales para delegar el cuidado, el efecto sobre su riqueza es, sin embargo, mayor. Es decir, se produce una "acumulación de (des)ventaja" (DiPrete y Eirich 2006) o un "efecto Mateo" (Merton 1968). Aunque en los casos de posiciones más aventajadas se destinan entre 1.000 y $2.500 €$ mensuales al cuidado, la riqueza familiar continúa manteniéndose o aumentando, dado que ninguno de los miembros de estas configuraciones familiares sale del mercado de trabajo. Sin em- bargo, en los casos de posiciones desaventajadas se interrumpe la carrera laboral, y el capital social y el escaso patrimonio de partida comienzan a reducirse.

Finalmente, las consecuencias de cuidar también varían en función del género. Los hombres de posiciones socioeconómicas elevadas realizan principalmente tareas de cuidado de tipo instrumental (gestión, organización...). Así, como acabamos de ver, el tener una posición de partida de cierto privilegio les lleva a atenuar consecuencias socioeconómicas de mayor grado. En los dos casos de estudio en posiciones sociales desventajadas, que realizan principalmente cuidados informales en el hogar, un cuidador principal es hombre y otra mujer. Sin embargo, mientras que en el caso de Manuel su entorno le atribuye cierto capital simbólico por el hecho de cuidar -estaría sacrificando la venta de su fuerza de trabajo al mercado laboral por realizar el cuidado en el hogar-, Toñi no adquiere esa recompensa simbólica por realizar la misma acción: ella "no estaría sacrificando nada", sino que estaría ocupando "su" lugar en el espacio social, un lugar subordinado.

Esta situación lleva a las mujeres de clases populares analizadas —doblemente desaventajadas - a alejarse de otros grupos sociales que no las "entenderían", replegándose sobre sí mismas, su hogar y sus iguales -esto es, mujeres de clases populares y cuidadoras de mayores dependientes-. El objetivo de este repliegue sobre sí mismas sería la búsqueda de espacios donde a su labor cotidiana se le atribuya valor y reconocimiento, la búsqueda de pares que se identifiquen en su situación y, por ende, estén dotadas de los esquemas cognitivos necesarios para poder reconocer lo "duro que es el cuidado de un familiar enfermo" y que no "le sigan la razón como a los locos" (Toñi, Caso 4, clase baja), como harían los que nunca han tenido que cuidar directamente y por mucho tiempo de un familiar dependiente severo.

\section{Discusión}

El impulso que lleva a cuidar, como consideraron investigaciones previas para el caso de España (Tobío 2013; Legarreta 2008), se basa en una reciprocidad sobre la que se articulan los cuidados: una devolución basada en una lógica de don-contra don. Sin embargo, este trabajo concluye que, aunque en todos los casos analizados los cuidadores recibieron cuidados -instrumentales y emocionales - en el pasado por parte de los que hoy son dependientes, la forma de devolución de dichos cuidados varía según la posición social. Mientras que en las clases bajas el contra don se mueve sobre el eje de la entrega, el sacrificio o el tiempo, en las altas se mueve sobre el eje de la gestión, el control o el dinero. Asimismo, posiciones sociales intermedias constituyen formas de devolución mixtas, estrategias híbridas y contra dones que utilizan recursos tanto temporales como 
económicos, dependiendo de cómo se combinen la gravedad de la enfermedad del dependiente, los recursos disponibles y las preferencias de cuidados.

En la misma línea, este trabajo encuentra similares resultados que Castelló (2011) en el sentido de que las clases más altas ejercen resistencia a la devolución del cuidado en forma de institucionalización. No obstante, a diferencia de dicha autora, en esta investigación encontramos que la resistencia a la institucionalización atraviesa por igual a las distintas clases sociales. Asimismo, hallamos que las clases altas no solo ejercen resistencia a la institucionalización, sino también al cuidado en el hogar. Es relevante observar, en este juego de resistencias, cómo la parte emocional del cuidado es la que encuentra mayores dificultades en su delegación - a una institución o a una cuidadora formal-, como concluyó Martinez-Buján (2008). Sin embargo, en el eje de clase encontramos un gradiente: mientras que en el caso de clase alta se delega a personas ajenas a la familia tanto el cuidado más instrumental como el más emocional, conforme se desciende en las posiciones la resistencia a delegar el trabajo emocional coge más fuerza. En el otro extremo, el caso de clase más baja, la delegación del cuidado a personas ajenas a la familia, tanto en su nivel emocional como instrumental, es rechazada, bajo la justificación de la desconfianza a personas ajenas a la familia. La delegación emocional del cuidado está, por tanto, estratificada socialmente.

Por su parte, Moré (2015) considera que las mujeres contratadas para realizar el cuidado terminan considerando a las personas dependientes que cuidan como "su propia gente". En esta investigación encontramos resultados parecidos, pero desde la perspectiva de los que contratan. En el caso de clase alta, éstos terminan considerando a las personas contratadas como "su gente". Asimismo, Moré (2015), desde el análisis de las personas que contratan, considera que éstas exaltan la parte emocional de los cuidados sobre la instrumental. En esta investigación se observa cómo las mujeres contratadas son valoradas por las personas que contratan tanto por su capacidad emocional como técnica, si bien se exalta con mayor intensidad la segunda. En este sentido, obtenemos resultados similares a los de Martínez-Buján (2008), pero desde el lado de la demanda. Así, la relación emocional entre cuidadora contratada y familiar contratante sería una relación de cariño "ficticia" por estar basada en una relación salarial. Sin embargo, con el paso del tiempo comienzan a establecerse vínculos de tipo emocional, sobrepasando lo laboral. No obstante, esta relación estaría basada en una interdependencia mutua, con intereses coordinados sobre el mantenimiento de dicha relación.

Respecto a la Ley de Dependencia, como se observó en la descripción de los casos, los recursos estatales son utilizados por las familias más como un añadido a las estrategias previas que como una modificación de las mismas. Estos recursos otorgan mayor flexibilidad y complejidad a las estrategias familiares en el cuidado a mayores, como también consideró Tobío (2013). No obstante, se observa cómo las familias aquí analizadas, ante las posibles opciones que da la ley, optan todas por la prestación económica. En todos los casos, el dinero de la prestación termina siendo un ingreso extra de las mujeres que ya realizaban el cuidado antes de la implantación de la ley en 2007, ya sean éstas contratadas o familiares. Así, como ya advirtieron investigaciones previas (Serrano, Artiaga y Dávila 2013; RodríguezCabrero 2011), la Ley de Dependencia reproduce el marcado carácter familista del Estado del Bienestar español, así como la desigualdad de género.

\section{Conclusión}

Este trabajo se centra en el análisis de los condicionantes que llevan a elegir una estrategia u otra, las tensiones y juegos de legitimidades que se manejan en cada una de las clases sociales y estrategias familiares y sus consecuencias para los cuidadores principales y familiares. Para ello se ha llevado a cabo una investigación etnográfica en hogares, realizando entrevistas cruzadas y observaciones.

De los análisis realizados se concluye que las elecciones que toman las familias en torno a la adopción de una estrategia u otra de cuidado es el resultado tanto de los recursos materiales y temporales de los que disponen las familias, como de la dimensión valorativa que se le atribuye a cada estrategia concreta en cada grupo social (por ejemplo, mientras que los individuos de clases altas entienden como razonable mercantilizar el cuidado, esta misma opción se observa con resistencia en las posiciones sociales desaventajadas). Asimismo, también son resultado de las condiciones de producción de los habitus de clase (Bourdieu 2007; Castelló 2011) y de las negociaciones en torno a la estrategia de cuidado ejercidas por los diferentes miembros de las configuraciones familiares.

Los términos sobre los que se categorizan los cuidados, así como las dimensiones de atribución de valor social, varían entre clases y entre géneros. La sumisión a la necesidad, las condiciones materiales y los juicios producidos por sus iguales inclina a las mujeres de clases populares hacia el rechazo de la compra de cuidados en el mercado, asumiendo un duro trabajo. Esta práctica no es una elección tomada sobre la base de la libertad de acción, sino que está constreñida por la estructura social; en efecto, es una elección mediada. En posiciones desaventajadas, los limites sobre la capacidad de acción que imponen las condiciones materiales de existencia terminan provocando el rechazo, bajo una percep- 
ción negativa, de otras estrategias de cuidado que no sean la de cuidar informalmente en el hogar, terminando por considerar esta última como el "buen cuidado". Asimismo, para las clases altas, el trabajo cotidiano de cuidados es relevado por la gestión del cuidado: estar al corriente de lo que ocurre, controlar a las personas contratadas o informarse sobre la enfermedad y su proceso. En estas posiciones sociales el "buen cuidado" se relaciona con la mercantilización del mismo, manteniéndolo en el hogar, pero bajo la contratación de cuidadoras remuneradas. Las oportunidades y constricciones materiales y las simbólicas terminan retroalimentándose entre sí y, en última instancia, colocan la dependencia como otro mecanismo clave en la acumulación de (des)ventaja social, económica y simbólica.

El impulso que lleva a cuidar a la persona dependiente se justifica con base en una devolución de un don recibido por parte de los cuidadores previamente. Sin embargo, las formas de devolución se caracterizan por ser principalmente materiales (dinero) en las posiciones sociales más elevadas y mayormente inmateriales (tiempo) en las posiciones más desaventajadas. Finalmente, las recompensas simbólicas por el hecho de realizar el trabajo de cuidados también llegan de forma desigual según el género. Mientras los hombres que son cuidadores principales son alabados por el hecho de serlo, esto no ocurre así con las mujeres.

Antes de terminar deben señalarse algunas limitaciones de este estudio. En primer lugar, es un estudio de carácter etnográfico, por lo que no se establecen asociaciones ni de correlación ni de causalidad entre las dimensiones analizadas, como son la clase social, las prácticas y estrategias familiares, la construcción del significado en torno al "buen cuidado" o las con- secuencias que sobre los recursos familiares tiene el cuidado de personas dependientes. En segundo lugar, al ser su material empírico entrevistas y observaciones, se atiende en mayor medida a las prácticas y, aunque se analizan discursos, se dejan de lado sus aspectos más generales, codificados y estereotipados, a los que se puede atender con mayor precisión usando técnicas como los grupos de discusión. Por último, el trabajo de campo se realizó en un entorno semiurbano - poblaciones de menos de 100.000 habitantes - y en una sola comunidad autónoma. Esto puede dejar de lado dinámicas propias de grandes urbes o diferencias regionales, ya que Andalucía se caracteriza por ser un modelo familista subvencionado (Martínez-Buján, 2014). Futuras investigaciones deberían ahondar en las limitaciones aquí señaladas.

\section{Agradecimientos}

Agradezco el apoyo y la cobertura que me ofrecieron los proyectos FEM2010-18827, financiado por el Ministerio de Ciencia e Innovación; y 4164391 - 8/10, financiado por el Instituto de la Mujer; ambos dirigidos por Amparo Serrano Pascual. Asimismo, agradezco los valiosos comentarios que me hicieron llegar Enrique Martín Criado, Marc Barbeta, Gloria Domínguez Alegría, Francisco Parada y, especialmente, Araceli Serrano. Esta investigación ha contado con la ayuda BES-2013-066777 de Formación de Personal Investigador financiada por el Ministerio de Economía y Competitividad. Una versión anterior de este trabajo fue galardonada con el primer premio en el IV Concurso de Jóvenes Sociólogos promovido por la Asociación Madrileña de Sociología y la UNED. La responsabilidad por los posibles errores que haya en el texto es exclusivamente atribuible al autor.

\section{NOTAS}

1. Las categorías originales son cinco: insignificante, leve, moderada, grave o total. Para la facilidad lectora hemos fusionado, a lo largo del artículo, las dos últimas categorías (grave y total) dentro de la categoría más amplia severa.

2. Existe consenso en la literatura en considerar al análisis de los cuidados el género como una cuestión central. No obstante, por razones de espacio aquí se presenta el análisis centrado en la clase social. Los análisis centrados en el género están disponibles bajo consulta al autor.

\section{ReferenCIAS BibLIOGRÁficas}

Abellán, A., C. Esparza y J. Pérez. 2011. "Evolución y estructura de la población en situación de dependencia". Cuadernos de Relaciones Laborales 29(1): 43-67. https:// doi.org/10.5209/rev_CRLA.2011.v29.n1.2

Andersson A, L. Levin y B. Emtinger. 2002. "The economic burden of informal care". International Journal of Techno Assess Health Care, 18(1): 46-54.

Becker, H. 2009. Trucos del oficio. Como conducir su investigación en Ciencias Sociales. Madrid: Siglo XXI.

3. Marga vive seis meses al año con ellos y seis meses en su hogar, en una localidad cercana, donde vive sola, pues no está casada ni tiene hijos.

4. Extraído del cuaderno de campo. Observación caso 2. Discusión entre las nietas. 29 de Noviembre de 2011.

5. Extraído del cuaderno de campo. Observación caso 3. Conversación con Manuel. 12 de Septiembre de 2011.

Bourdieu, P. 2007. El sentido práctico. Buenos Aires: Siglo XXI.

Bourdieu, P. 2012. La distinción. Criterios y bases sociales del gusto. Madrid: Taurus.

Carrasco, C. 2013. "El cuidado como eje vertebrador de una nueva economía". Cuadernos de Relaciones Laborales 31: 3956. https://doi.org/10.5209/rev_CRLA.2013.v31.n1.41627

Castelló, L. 2011. "La gestió quotidiana de la cura. Una qüestió de gènere i classe". Tesis doctoral. De- 
partment de Sociologia, Universitat Autònoma de Barcelona. Barcelona.

Conde, F. 2009. Análisis sociológico del sistema de discursos. Madrid: CIS.

DiPrete, T. y G. Eirich. 2006. "Cumulative advantage as a mechanism for inequality: A review of theoretical and empirical developments." Annual Review of Sociology 32: 271-297.

Durán, M.A. 2000. Los costes invisibles de la enfermedad. Bilbao: BBVA.

Elías, N. 1987. La soledad de los moribundos. Madrid: Fondo de Cultura Económica.

Esping-Andersen, G. 1990. The three worlds of welfare capitalism. Cambridge: Polity Press.

Fernández-Carro, C. 2016. "Ageing at home, co-residence or institutionalisation? Preferred care and residential arrangements of older adults in Spain". Ageing \& Society 36: 586-612. https://doi.org/10.1017/ S0144686X1400138X

Folbre, N., 2007. Valuing Children: Rethinking the economics of the family. Cambridge MA: Harvard University press.

Godelier, M. 2000. "Acerca de las cosas que se dan, de las cosas que se venden y de las que no hay que vender ni dar sino que hay que guardar. Una reevaluación crítica del 'Ensayo Sobre el Don' de Marcel Mauss”. Revista Hispania 204: 11-26.

Ibáñez, J. 1979. Más allá de la sociología. El grupo de discusión: técnica y crítica. Madrid: Siglo XXI.

Legarreta, M. 2008. "El tiempo donado en el ámbito doméstico. Reflexiones para el análisis del trabajo doméstico y de cuidados". Cuadernos de Relaciones Laborales 26: 49-73.

Lombardo, E. y M. León. 2014. "Políticas de igualdad de género y sociales en España: origen, desarrollo y desmantelamiento en un contexto de crisis económica". Investigaciones Feministas 5: 13-35.

Martín Criado, E. 1998. "Los decires y los haceres". Papers. Revista de Sociología 56: 57-71. https://doi. org/10.5565/rev/papers. 1944

Martín Criado, E., C. Gómez Bueno y A. Fernández. 2000. Familias de Clase Obrera y Escuela. Donostia: Iralka.

Martín Criado, E. 2014. "Mentiras, inconsistencias y ambivalencias. Teoría de la acción y análisis del discurso". Revista Internacional de Sociología 72: 116-138. https://doi.org/10.3989/ris.2012.07.24

Martín Palomo, M.T. 2013. "Tres generaciones de mujeres, tres generaciones de cuidados. Apuntes sobre una etnografía moral". Cuadernos de Relaciones Laborales 31: 115-138. https://doi.org/10.5209/rev_CRLA.2013. v31.n1.41641

Martín, S. y C. Vilaplana. 2012. "Trade-off between formal and informal care in Spain". The European Journal of Health Economics 13(4): 461:490. https://doi. org/10.1007/s10198-011-0317-z

Martínez Buján, R. 2008. Bienestar y cuidados. El oficio del cariño. Mujeres inmigrantes y mayores nativos. Tesis doctoral. La Coruña: Departamento de Sociología, Universidad de La Coruña.

Martínez Buján, R. 2014. "Los modelos territoriales de organización social del cuidado a personas mayores en los hogares". Revista Española de Investigaciones Sociológicas 145: 99-126.
Mauss, M. 1979. Sociología y Antropología. Madrid: Tecnos.

Merton, R. K. 1968. "The Mathew Effect in Science". Science 159 (3810): 56-63. https://doi.org/10.1126/science. 159.3810 .56

Moré, P. 2015. La organización social de los cuidados a personas mayores en Madrid y París: de la domesticidad a la profesionalización en la intersección género, clase y etnicidad. Tesis doctoral. Madrid: Departamento de Sociología III, Universidad Complutense de Madrid.

Otero, A., M.J. García de Yebenes, A. Rodríguez-Laso y M. Zunzunegui. 2003. "Unmet home care needs among community-dwelling elderly people in Spain". Aging Clin Exp Res 15(3): 234-242. https://doi.org/10.1007/ BF03324504

Pérez Orozco, A. 2014. Subversión feminista de la economía. Aportes para un debate sobre el conflicto capital-vida. Madrid: Traficantes de sueños.

Prieto, C. y Serrano, A. 2013. "Los Cuidados entre el trabajo y la vida". Cuadernos de Relaciones Laborales 31(1): 11-16.

Rodríguez Cabrero, G. 2011. "Políticas sociales de atención a la dependencia en los regímenes de bienestar de la Unión Europea". Cuadernos de Relaciones Laborales 29: 13-42. https://doi.org/10.5209/rev_CRLA.2011. v29.n1.1

Rogero, J. 2009. "Distribución en España del cuidado formal e informal a las personas de 65 años y más en situación de dependencia". Revista Española de Salud Pública 83: 393-405. https://doi.org/10.1590/S113557272009000300005

Rogero, J. 2010. Los tiempos del cuidado: El impacto de la dependencia de los mayores en la vida cotidiana de sus cuidadores. Madrid: IMSERSO.

Rogero, J., M. Prieto y M. Rosenberg. 2008. "Health services use by older people with disabilities in Spain: do formal and informal care matter?" Ageing \& Society 28(7): 959978. https://doi.org/10.1017/S0144686X08007381

Rollins, J. 1985. Between women: Domestics and their employers. Philadelphia: Temple University Press.

Sennet, R. y J. Cobb. 1973. The hidden injuries of class. New York: Knopf.

Serrano, A., A. Artiaga y M. Dávila. 2013. "Crisis de los cuidados, Ley de Dependencia y confusión semántica". Revista Internacional de Sociología 71(3): 669-674. https://doi.org/10.3989/ ris.2012.07.30

Spijker, J. y P. Zueras. 2016. "El cuidado a los mayores en un contexto de envejecimiento y cambio social, político y económico". Panorama Social, 23: 109-129.

Thomas, C. 2011. "Deconstruyendo los conceptos de cuidados". En El trabajo de cuidados. Historia, teoría y políticas, editado por C. Carrasco, C. Borderías, T. Torns. Madrid: La Catarata.

Tobío, C. (2013). "Estado y familia en el cuidado de las personas: Sustitución o complemento". Cuadernos de Relaciones Laborales 32(1): 17-38

Wilkin, D. (1987): "Conceptual problems in dependency research". Social Science and Medicine 24(10): 867873. https://doi.org/10.1016/0277-9536(87)90188-2 
TOMÁS CANO es Licenciado en Sociología (Universidad de Granada) y Máster en Metodología de la Investigación en Ciencias Sociales (Universidad Complutense de Madrid). Actualmente es Doctorando en la Universitat Pompeu Fabra y Research Fellow del ARC Centre of Excellence for Children and Families over the Life Course. Su tesis doctoral estudia bajo qué condiciones los hombres se implican en los cuidados, y qué efectos tiene dicha implicación sobre el desarrollo cognitivo y emocional de los niños. Para ello usa datos de uso del tiempo longitudinales y transversales de Australia y España. Ha realizado estancias de investigación en el German Institute for Economic Research (DIW-Berlin), University College of London (UK) y en la University of Queensland (Australia). www.tomascano.eu 